\title{
High - Sensitivity C - Reactive Protein: An Independent Proinflammatory Cardiac Marker in Healthy Overweight and Obese Individuals
}

\author{
B. Asegaonkar Shilpa ${ }^{1}$, S. Bavikar Jayashree ${ }^{2}$, Marathe Amruta ${ }^{3}$, Tekade Mangesh ${ }^{4}$ \\ and N. Asegaonkar Balaji ${ }^{5}$ \\ ${ }^{1}$ Course in Clinical Nutrition, Department of Biochemistry, Government Medical College \\ Aurangabad, Maharashtra India \\ ${ }^{2}$ Department of Biochemistry, Government Medical College Aurangabad, Maharashtra India \\ ${ }^{3}$ Department of Biochemistry, L.T.T.M. Medical College, Mumbai, Maharashtra India \\ ${ }^{4}$ Department of Biochemistry, I.G.M.C.Nagpur, Maharashtra India \\ ${ }^{5}$ Cardiac Anaesthesiology, Department of Anaesthesiology, M.G.M. College Aurangabad \\ Maharashtra Aurangabad, India
}

Correspondence should be addressed to: B. Asegaonkar Shilpa; b_asegaonkar@yahoo.com

Received Date: 25 September 2013; Accepted Date: 14 December 2013; Published Date: 27 March 2014

Academic Editor: Bernard Man Yung Cheung

Copyright (C) 2014 B. Asegaonkar Shilpa, S. Bavikar Jayashree, Marathe Amruta, Tekade Mangesh and N. Asegaonkar Balaji. Distributed under Creative Commons CC-BY 3.0

\begin{abstract}
In Asian countries, Indians are at high risk of premature CVD and T2DM due to abdominal obesity. Adipose tissue over the abdomen is a major source of inflammatory mediators. Numerous studies have demonstrated role of serum high sensitivity C reactive protein (hsCRP) as independent predictor of metabolic syndrome, T2DM and atherosclerotic diseases among healthy individuals. We postulated that in spite of normal glucose tolerance and normal lipid profile, obese persons are at high cardiac risk due to chronic low grade systemic inflammation.

In present cross sectional study we enrolled 50 metabolically healthy overweight and obese individuals and 50 age and sex matched lean controls. We recorded history, anthropometric measures of adiposity BMI, waist and hip circumference, in study group. After overnight fast blood samples were assayed for blood glucose, lipid profile and hsCRP levels.

Serum hsCRP levels were raised significantly in cases compared to control (mean 3.8+/-1.8 Vs $1.2+/-0.7 \mathrm{p}<0.001$ ). We found significant positive association of hsCRP with anthropometric indices BMI and waist: hip ratio. In obese group there was evidence of significant family history of obesity, T2DM and CVD in first degree relatives compared to control.
\end{abstract}

Cite this Article as: B. Asegaonkar Shilpa, S. Bavikar Jayashree, Marathe Amruta, Tekade Mangesh and N. Asegaonkar Balaji (2014), "High - Sensitivity C - Reactive Protein: An Independent Proinflammatory Cardiac Marker in Healthy Overweight and Obese Individuals," Journal of Research in Obesity, Vol. 2014 (2014), Article ID 731358, DOI: 10.5171/2014.731358 
Our results suggests strong link between indices of adiposity and elevated hsCRP. Obesity without any measurable metabolic abnormality is a state of chronic low grade systemic inflammation. Serum hsCRP is an inexpensive, simple tool to predict cardiac risk in apparently individuals at early stage. This high risk group should be targeted for therapeutic lifestyle changes to prevent further sequels.

Keywords: High sensitivity $\mathrm{C}$ reactive protein, overweight, obesity, cardiovascular risk.

\section{Introduction}

\section{Background of Study}

Obesity is a multifactorial complex progressive disorder of chronic energy surplus when energy intake exceeds energy expenditure leading to accumulation of excess of adipose tissue. In last two decades prevalence of obesity has been tripled resulting in its devastating consequences like type 2 diabetes mellitus (T2DM), hypertension, dyslipedemia, cardiovascular disease (CVD), stroke, sleep apnea syndrome and some malignancies.

Worldwide more than $400 \times 10^{6}$ adults are obese and this number is expected to double by 2015 due to unhealthy lifestyle like excessive dietary fat intake, physical inactivity and genetic susceptibility (Jennifer et al 2012). This upsurge in obesity in both developed and developing countries is responsible for increased burden of noncommunicable diseases. In developed world $2-7 \%$ of total healthcare cost is attributed to obesity and its complications. Nowadays obesity is the fifth most common cause of disease burden. It has been stated that obese persons are five times prone to develop hypertension compared to lean persons. Also in $90 \%$ of cases of T2DM obesity is leading cause for Insulin Resistance which is precedent of not only cardiovascular disease but also chronic kidney disease (IDF Diabetes Atlas 2003).

\section{Research Motivation}

Asian Indians are at high risk of premature CVD and T2DM due to abdominal obesity. In India, six fold rises in prevalence of obesity in next decade is predicted. Obesity, a precursor of several noncommunicable diseases predisposes to metabolic syndrome, impaired glucose tolerance and closely linked with T2DM and CVD. (Misra and Khurana 2008).

Previously adipose tissue was considered as a passive storage of fat depots playing central role in lipid metabolism. But now its role as major source of inflammatory mediators has been well recognized. About $25 \%$ of proinflammatory cytokines Interleukin- 6 is synthesized in adipose tissues and released in circulation which triggers release of acute phase reactant $C$ Reactive Protein (CRP) from hepatocytes. Thus obesity is a state of low grade chronic inflammation which is one of the important contributors for T2DM and atherogenesis. (Jeemon et al 2011)Now major role of inflammation in causation, progression and complications of atherosclerosis has been well accepted.

\section{Literature Review}

High sensitivity C Reactive Protein (hsCRP), a pentameric protein from Pentraxin family is a golden proinflammatory and proatherogenic marker. It is easily measured and widely investigated prototypic inflammatory biomarker (Jialal and Devaraj 2001). Also it adds predictive power to current coronary risk factors.

In obese individuals adipocytes express release of proinflammatory cytokine IL-6 stimulating hepatic production of hsCRP, a sensitive marker of systemic inflammation. Numerous studies have demonstrated role of hSCRP as predictor of metabolic syndrome, T2DM and atherosclerotic diseases among healthy individuals (Ridker et al 2004, 2008, Kelishadi et al 2009).

Jeemon et al (2011) studied association of hsCRP with traditional cardiac risk factors in Indians and observed that indices of adiposity correlate well with hsCRP levels and hence are surrogate markers of inflammatory status. More than $50 \%$ of 
coronary events have been observed in apparently healthy individuals without any cardiac risk factor including dyslipedemia. So researchers started to search for other risk factors for global cardiac risk prediction. Among the emerging biomarkers, hsCRP is widely investigated and accepted promising inflammatory risk predictor in healthy individuals. (Michael B. Clearfield, DO 2005)

\section{Research Question}

We postulated that in spite of normal glucose tolerance and normal lipid profile obese persons are at high cardiac risk due to chronic low grade systemic inflammation. With this perspective we planned present study to investigate a state of chronic low grade systemic inflammation by measuring serum hsCRP levels in metabolically healthy overweight and obese individuals and compare with a group of age and sex matched lean controls.

\section{Research Design and Methodology}

Present cross sectional study was conducted as per the guidelines of Institutional Ethical Committee. After screening for glucose tolerance and lipid profile, out of 112 individuals 50 healthy overweight and obese individuals were selected as cases (BMI $\left.>25 \mathrm{Kg} / \mathrm{m}^{2}\right)$ and 50 healthy age and sex matched individuals with normal weight as controls (BMI < $24.9 \mathrm{Kg} / \mathrm{m}^{2}$ ). Informed written consents were obtained from all subjects. Patients with metabolic syndrome, cardiovascular disease, hypertension, T2DM, dyslipedemia, acute systemic illness, liver diseases, chronic obstructive pulmonary disease, and arthritis were excluded. Also patients on anti-inflammatory drugs, lipid lowering drug therapy, oral contraceptive pills and hormone replacement therapy were excluded from study group. A standard questionnaire was designed to record demographic data and personal habits including age, sex, and other co morbid conditions, history of CVD and T2DM in first degree relatives and history of ingestion of anti inflammatory drugs within last ten days. Details of smoking history, alcohol use, physical activity, social class were recorded. Systolic and diastolic blood pressures were recorded for all subjects.

For anthropometric measurements, we recorded weight (kilograms), height (centimeters), waist circumference (centimeters), hip circumference (centimeters) by standardized techniques. BMI was calculated as weight (kilograms)/ height (meters ${ }^{2}$ ) as a measure of obesity and waist: hip ratio as an index of abdominal adiposity. Cut off points for obese Asian women were $\mathrm{BMI}>25 \mathrm{~kg} / \mathrm{m} 2$ waist $>80 \mathrm{~cm}, \quad W H R>0.88$ as per WHO standards for Asians. Cut off points for obese Asian men were BMI $>25 \mathrm{~kg} / \mathrm{m} 2$ waist $>85 \mathrm{~cm}$, WHR $>0.8$ (Snehalatha 2003).

All the study subjects were recalled for assessment of biochemical parameters. After overnight fast, $5 \mathrm{ml}$ venous blood samples were collected and after serum separation various biochemical assays were performed. In individuals with normal blood glucose level and lipid profile, serum hsCRP levels were assayed. Blood glucose (Glucose Oxidase Peroxidase end point method), serum total cholesterol (TC- Cholesterol Oxidase Peroxidase), serum triglycerides (TGLipase/Glycerokinase/Glycerophosphate oxidase) and High Density Lipoproteins (HDL- direct method-polyethylene glycolpretreated enzymes) were assayed using fully automatic analyzer from Transasia. Serum Low Density Lipoprotein (LDL) was estimated by Friedewalds formula. Serum hsCRP was estimated by chemiluminescence's immunoassay using Acculite CLIA microwells with Assay kits from Monobind INC, Lake forest, CA 92630 USA.

Statistical analysis was performed using the SPSS data analysis system. Demographic and biochemical data of all subjects was analyzed as mean+/-S.D.and student' $t$ ' test used to demonstrate the significance of different variables. $P$ values less than 0.05 were considered as significant, less than 0.001 as highly significant and more than 0.05 as nonsignificant. Pearson's correlation analysis was carried out to determine the 
correlation of hsCRP ( $\mathrm{r}$ values) with various anthropometric measures in study group.

\section{Results}

Demographic characteristics of cases and controls are represented in table 1.

Table 1: Demographic Data of Study Subjects

\begin{tabular}{|c|c|c|c|}
\hline Variable & $\begin{array}{l}\text { Cases (Overweight } \\
n=16 \text { and obese } n=32 \text { ) } \\
\text { Mean+/- S.D. }\end{array}$ & $\begin{array}{l}\text { Control } n=50 \\
\text { Mean+/- S.D. }\end{array}$ & P value \\
\hline Age & $32.5+/-6.4$ & $34.3+/-5.3$ & 0.65 \\
\hline $\operatorname{Sex}(M / F)$ & $32: 18$ & $29: 21$ & \\
\hline BMI & $28.3+/-3.4$ & $21.2+/-2.9$ & $<0.001^{* *}$ \\
\hline Waist circumference & $101.3+/-9$ & $83+/-2.5$ & $<0.001^{* *}$ \\
\hline Waist : Hip & $1.3+/-0.3$ & $0.82+/-0.2$ & $<0.001^{* *}$ \\
\hline Smokers & 6 & 4 & 0.32 \\
\hline Alcoholics & 8 & 6 & 0.34 \\
\hline $\begin{array}{lll}\text { Family history of } \\
\text { Obesity }\end{array}$ & 34 & 11 & $<0.001^{* *}$ \\
\hline $\begin{array}{l}\text { Family history of } \\
\text { T2DM }\end{array}$ & 27 & 18 & $<0.05^{*}$ \\
\hline Family history of CVD & 10 & 5 & $<0.05^{*}$ \\
\hline $\begin{array}{l}\text { Systolic blood } \\
\text { pressure }(\mathrm{mm} / \mathrm{Hg})\end{array}$ & $124+/-21$ & $112+/-10$ & 0.42 \\
\hline $\begin{array}{l}\text { Diastolic blood } \\
\text { pressure }(\mathrm{mm} / \mathrm{Hg})\end{array}$ & $93+/ 8$ & $75+/-6$ & 0.32 \\
\hline
\end{tabular}

$\mathrm{P}<0.001^{* *}$ - highly significant $\mathrm{P}<0.05^{*}$ - significant

Table 2: Biochemical Parameters among Cases and Controls

\begin{tabular}{|c|c|c|c|}
\hline $\begin{array}{l}\text { Biochemical } \\
\text { Parameters }\end{array}$ & Cases & Controls & P Value \\
\hline Blood glucose mg/dl & $98.5+/-10$ & $76+/-12$ & $<0.05^{*}$ \\
\hline $\begin{array}{l}\text { Serum total } \\
\text { cholesterol mg/dl }\end{array}$ & $147+/-13$ & $138+/-10$ & 0.06 \\
\hline $\begin{array}{l}\text { Serum triglycerides } \\
\mathrm{mg} / \mathrm{dl}\end{array}$ & $167+/-18$ & $102+/-23$ & $<0.05^{*}$ \\
\hline Serum HDL mg/dl & $57+/-8$ & $59+/-7$ & 0.061 \\
\hline Serum LDL mg/dl & $115+/-12$ & $92+/-8$ & $<0.05^{*}$ \\
\hline Serum hsCRP mg/dl & $3.8+/-1.8$ & $1.2+/-0.7$ & $<0.001^{* *}$ \\
\hline
\end{tabular}

Table 3. Pearson Correlation Analysis (R Values) Of Hscrp with Anthropometric Measures in Cases

\begin{tabular}{|l|l|l|l|}
\hline & BMI & W/ H & hsCRP \\
\hline BMI & - & 0.21 & 0.26 \\
\hline W/ H & & - & $0.53^{*}$ \\
\hline
\end{tabular}

Correlation analysis was performed for two measures of obesity BMI and waist: hip ratio with serum hsCRP levels. Waist: hip ration has significant positive correlation with hsCRP than that of BMI. Following scatter plot graph shows hsCRP as a function of waist: hip ratio. 


\section{hsCRP}

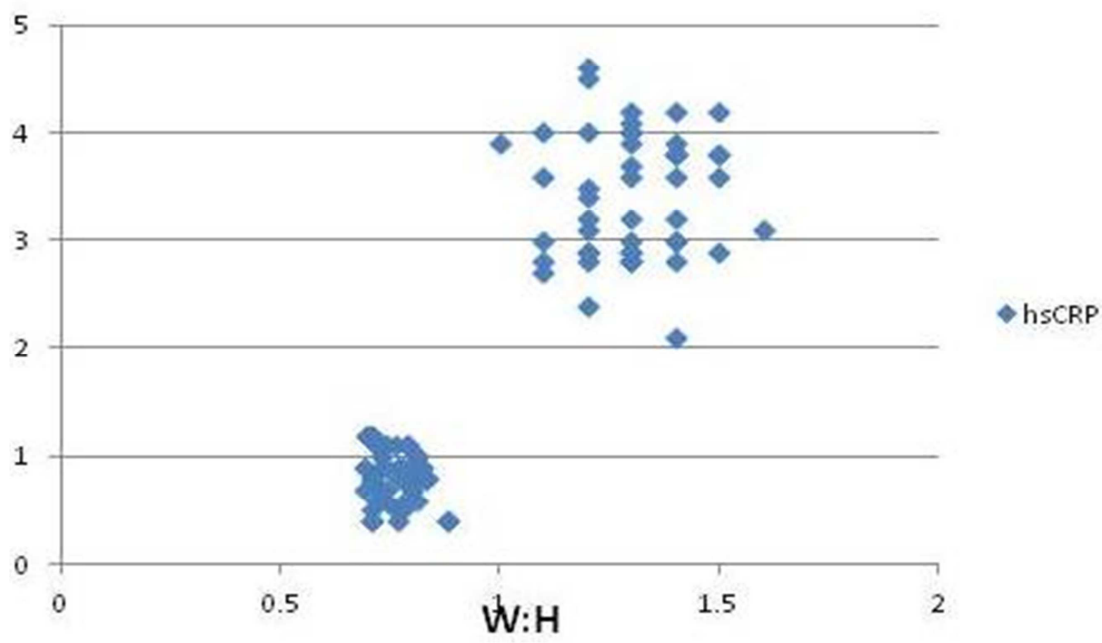

\section{Discussion}

In the present cross sectional study we studied serum hsCRP, a proinflammatory prototypic cardiac risk marker in overweight and obese subjects without any metabolic abnormality. Serum hsCRP levels were raised significantly in cases compared to control (mean 3.8+/-1.8 Vs $1.2+/-0.7 \mathrm{p}<$ 0.001). We found significant positive association of hsCRP with anthropometric indices BMI and waist: hip ratio. In obese group there was evidence of significant family history of obesity, T2DM and CVD in first degree relatives compared to control.

Our results suggests strong link between indices of adiposity and elevated hsCRP. Visceral adipose tissue, a source of proinflammatory cytokines triggers synthesis of CRP from liver. In obese individuals, adipose tissue $\mathrm{T}$ cells release cytokines contributing to development of Insulin resistance. Chronic low grade systemic inflammation and Insulin resistance in combination further leads to deranged metabolic profile. Raised hs-CRP levels in obesity reflects the enhanced production of cytokines which may render otherwise stable atherosclerotic plaques unstable and thus susceptible to rupture (Eckel et al 2002).

Our data confirm findings of prior studies among Indians showing elevated levels of hsCRP correlating with measures of adiposity in healthy individuals (S.K.Sharma et al 2008, N. Dev and S Marcus 2012, Chandorkar et al 2011). K. Gokulkrishnan et al (2009) in their CURE 64 study observed significant association between systemic inflammation measured by hsCRP and leukocyte count with cardiac risk factors in persons with normal glucose tolerance. Karelis et al (2005) investigated inflammatory state in women with metabolically healthy but obese (MHO) phenotype. They observed favorable inflammatory state with low hsCRP and $\alpha 1$ antitrypsin in MHO group compared to insulin resistant group. Serum hsCRP is an inexpensive tool to predict development of T2DM, metabolic syndrome, CVD in not only healthy adults but in adolescents and children also (Leandro Soriano-Guille'n et al 2008, HH El-shorbagy and IA Ghoname 2010).

Data from Indian Atherosclerosis Research Study by Veena Rao et al (2010) indicated role of high levels of hsCRP as additive tool to identify CAD affected high risk subjects who can develop recurrent coronary event in future. They observed that individuals in top quartile of hsCRP have threefold excess risk of reinfarction even after adjustment for traditional risk factors. It is a novel biomarker with potential to include in risk prediction model due to its nature of stability, low diurnal variation and ease to measure. 
We investigated overweight and obese subjects for serum hsCRP level before worsening of metabolic profile. So this high risk population should be targeted for therapeutic lifestyle change so as to prevent further complications of obesity. Addition of hsCRP estimation to assess risk improves global risk stratification irrespective of value of LDL cholesterol.(Jialal and Devaraj 2009 ). Serum hs-CRP is a promising biomarker to stratify risk particularly in intermediate group who accounts for more than 70\% vascular events. It is linked with pathophysiology of atherogenesis due its association with insulin resistance, leptin and cytokine function, endothelial dysfunction and platelet inhibition (Paul Ridker 2008).

Serum hsCRP levels can be reduced with lifestyle modifications and therapeutic interventions. Beavers KM and Nicklas BJ (2011) assessed six large studies on effect of lifestyle interventions on inflammatory markers in their review. They summarized successful role of dietary changes on improvement in inflammatory and metabolic biomarkers. Heilbronn LK (2001) reported reduction in hsCRP by $26 \%$ in healthy obese women after very low fat diet for 12 weeks. Jupiter trial (Ridker PM et al 2008) interpreted beneficial role of statin therapy in healthy individuals without any risk factors to reduce hsCRP levels which in turn helps in primary prevention of CVD.

Obesity is a proinflammatory and prothrombotic state before impairment of metabolic profile. Obese people without overt cardiac risk factors like hypertension, T2DM and dyslipedemia may remain under false impression of being healthy. Hence additional screening of these apparently healthy individuals with adjuvant novel biomarker hsCRP will help in global cardiovascular risk assessment. Weight reduction by dietary and exercise intervention remains an important cornerstone to improve long term cardiovascular risk before development of metabolic syndrome.

\section{Conclusion}

In conclusion, overweight and obese persons without any measurable metabolic abnormality are in a state of chronic low grade inflammation. Serum hsCRP is an inexpensive, simple tool to predict cardiac risk in apparently individuals at early stage. This high risk group should be targeted for therapeutic lifestyle changes to prevent further sequels.

\section{Study Limitations}

Our study has limitations due to small number of sample size and single measurement of biochemical parameters. Being cross sectional, present work cannot study cause and effect relation of hsCRP with obesity. In future large population based prospective interventional studies are needed to identify high risk subjects and minimize their risk.

\section{References}

Anoop Misra \& Lokesh Khurana (2008). "Obesity and the Metabolic Syndrome in Developing Countries," Journal of Clinical Endocrinology and Metabolism, 93(11):S9 S30

Antony D. Karelis, May Faraj, Jean-Philippe Bastard, David H. St-Pierre, Martin Brochu, Denis Prud'homme \& Remi RabasaLhoret (2005). "The Metabolically Healthy but Obese Individual Presents a Favorable Inflammation Profile," The Journal of Clinical Endocrinology \& Metabolism, 90(7):4145-4150.

Beavers, K. M. \& Nicklas, B. J. (2011). "Effects of Lifestyle Interventions on Inflammatory Markers in the Metabolic Syndrome," Frontiers in Bioscience (Scholar Edition), 1(3): 168-77.

Chamukuttan

Snehalatha, Vijayviswanathan \& Ambady Ramachandran (2003). "Cutoff Values for Normal Anthropometric Variables in Asian Indian Adults," Diabetes Care, 26:13801384. 
Eckel, R. H., Barouch, W. W. \& Ershow, A. G. (2002). "Report of the National Heart, Lung, and Blood Institute-National Institute of Diabetes and Digestive and Kidney Diseases Group on the Pathophysiology of Obesity-Associated Cardiovascular Disease," Circulation,105:2923-2928.

Gokulakrishnan, K., Deepa, R., Sampathkumar, R., Balasubramanyam, M. \& Mohan. V. (2009). "Association of Leukocyte Count and HsCRP with Metabolic Abnormalities in Subjects with Normal Glucose Tolerance (CURES - 64)," 7(3): 205-10.

Hatem Hamed El-Shorbagy \& Ibraheim Abdel-Aziz Ghoname (2010). "HighSensitivity C-Reactive Protein as a Marker of Cardiovascular Risk in Obese Children and Adolescents HEALTH 2," 1078-1084.

Heilbronn, L. K., M. Noakes \& P. M. Clifton (2001). "Energy Restriction and Weight Loss on Very-Low Fat Diets Reduce C Reactive Protein Concentrations in Obese, Healthy Women," Arteriosclerosis, Thrombosis and Vascular Biology, 21:881883.

International Diabetes Federation. (2003). Diabetes Atlas (second edition) 4 [http://www.worlddiabetesfoundation.or $\mathrm{g} /$ composite-35.htm].

Ishwarlal Jialal \& Sridevi Devaraj (2001). "Inflammation and Atherosclerosis the Value of the High-Sensitivity C-Reactive Protein Assay as a Risk Marker," American Journal of Clinical Pathology, 116 (Suppl 1):S108-S115

Jialal, I. \& Devaraj, S. (2009). "Jupiter to Earth: CRP Promotes Atherothrombosis," Metabolic Syndrome and Related Disorders, 7(1): 1-3.

Leandro Soriano-Guillén, Bárbara Hernández-García, Jimena Pita, Nieves Domínguez-Garrido, Genoveva Del RíoCamacho \& Adela Rovira (2008). "HighSensitivity C-Reactive Protein is a Good Marker of Cardiovascular Risk in Obese Children and Adolescents," European Journal of Endocrinology, 159 R1-R4
Michael, B. \& Clearfield, D. O. (2005). "CReactive Protein: A New Risk Assessment Tool for Cardiovascular Disease," The Journal of the American Osteopathic Association, 105 (9) 409-16.

Nirmitha Dev \& Sara Rani Marcus (2012). "High Sensitive C-Reactive Protein, an Independent and Early Novel Inflammatory Marker in Healthy Obese Women," Biomedical Research 23 (1): 73-77

Panniyammakal Jeemon, Dorairaj Prabhakaran, Lakhmy Ramakrishnan, Ruby Gupta, Ahmed, F., Thankappan, K. R., Kartha, C. C., Vivek Chaturvedi, Reddy, K. S. and the Sentinel Surveillance in Industrial Populations Study Group (2011). "Association of High Sensitive C-Reactive Protein (Hscrp) with Established Cardiovascular Risk Factors in the Indian Population," 8: 1-8.

Ridker, P. M. (2008). "High-Sensitivity CReactive Protein as a Predictor of All-Cause Mortality: Implications for Research and Patient Care," Clinical Chemistry 54:2 234237

Ridker, P. M., Danielson, E., Fonseca, F., Genest, J., Gotto, A., Kastelein, J. et al. (2008). "Rosuvastatin to Prevent Vascular Events in Men and Women with Elevated CReactive Protein," The New England Journal of Medicine, 359:2195- 2207.

Ridker, P. M., Wilson, P. W. F. \& Grundy, S. M. (2004). "Should C-Reactive Protein be Added to Metabolic Syndrome and to Assessment of Global Cardiovascular Risk?," Circulation, 109: 2818-2825.

Roya Kelishadi, Mohsen Sharifi, Alireza Khosravi \& Khosrow Adeli (2007). "Relationship between C-Reactive Protein and Atherosclerotic Risk Factors and Oxidative Stress Markers among Young Persons 10-18 Years Old," Clinical Chemistry 53:3;456-464

Sharma, S. K., Hemant Kumar Mishra, Hanish Sharma, Ashish Goel, Sreenivas, V., Vinay Gulati \& Mohammad Tahir (2008). "Obesity, and Not Obstructive Sleep Apnea, is Responsible for Increased Serum Hs-CRP Levels in Patients with Sleep-Disordered 
Breathing in Delhi," Sleep Medicine; 9:14956.

Shea, J., Diamandis, E. P., Sharma, A. M., Després, J. P., Ezzat, S. \& Greenway, F. (2012). "The Obesity Epidemic," Clinical Chemistry, 58(6) 968-973.
Suneeta Chandorkar, Neha Vaidya \& Ruchi Patel (2011). "Anthropometric Indices, Lipid Profile and Hs Crp Levels in Adults (25-60 Years) in an Urban Setting International," Journal of Applied Biology and Pharmaceutical Technology, 2(3) 4-30. 